\title{
ARTí́culo
}

\section{Circulación residual en el fiordo Aysén, Chile, en condiciones de primavera}

\author{
Residual circulation in Aysén Fjord, Chile, \\ during spring conditions \\ Julio Salcedo-Castro ${ }^{1}$, Raúl Cruz-Gómez ${ }^{2}$, Andry W. \\ Ratsimandresy $^{3}$ y Natália Pillar da Silva ${ }^{4}$
}

\begin{abstract}
${ }^{1}$ Centro de Estudios Avanzados, Universidad de Playa Ancha, Traslaviña 450, Viña del Mar, Chile. julio.salcedo@upla.cl ${ }^{2}$ Departamento de Física, Universidad de Guadalajara, Blvd. Marcelino García Barragán y Calzada Olímpica, C.P. 44840, Guadalajara, Jalisco, México

${ }^{3}$ Northwest Atlantic Fisheries Centre, Department of Fisheries and Oceans, St. John's, NL A1C 5X1, Canada

${ }^{4}$ Departamento de Ciências Atmosféricas, Instituto de Astronomia, Geofísica e Ciências Atmosféricas, Universidade de São Paulo, Rua do Matão, 1226, 05508-900, São Paulo, SP, Brasil
\end{abstract}

\begin{abstract}
Time series of currents speed from 3 current meters moored at the mouth of Aysén Fjord, between October and November 1996, were analyze to estimate residual circulation and to relate surface flow to variations in salinity, wind and Aysén River runoff. Residual circulation and salinity at $6 \mathrm{~m}$ were determined by events of strong river discharge and relaxing of local wind. Periods of strong outflow and evident drop of salinity at the mouth of the fjord were preceded by a weakening of wind and in some cases were accompanied by an increase of Aysén river discharge. Moreover, the results confirm the 3-layer structure proposed for Aysén fjord, since seaward average currents were observed at 6 and $110 \mathrm{~m}$ depth, whereas weak transition average currents were observed at $50 \mathrm{~m}$ depth, representing a transition between an intermediate landward flow and the seaward bottom layer.
\end{abstract}

Key words: Fjord, estuary, stratification, sound, river, sill

\begin{abstract}
Resumen.- Se analizaron las series de tiempo de velocidad de la corriente en un anclaje de 3 correntómetros instalados en la boca del fiordo Aysén, entre octubre y noviembre de 1996, obteniendo la circulación residual y relacionando el flujo superficial con la variación de la salinidad, el viento y el caudal del río Aysén. Se observó que la circulación residual y la salinidad a 6 m, estuvieron determinadas por eventos de fuerte descarga del río y relajamiento del viento local. Periodos de fuerte flujo y un acentuado descenso de salinidad en la boca del fiordo estuvieron precedidos por una disminución del viento y en algunos casos estuvieron acompañados de aumentos en el caudal del río Aysén. Por otra parte, los resultados obtenidos confirman la estructura de 3 capas propuesta para el fiordo Aysén, ya que a 6 y $110 \mathrm{~m}$ de profundidad se observaron velocidades promedio de salida, mientras que a $50 \mathrm{~m}$ se registraron débiles corrientes con un flujo promedio también de salida y que correspondería a la transición entre una capa intermedia de ingreso hacia el fiordo y la capa profunda de salida.
\end{abstract}

Palabras clave: Fiordo, estuario, estratificación, seno, río, umbral

\section{INTRODUCCIÓN}

Los fiordos se definen como estructuras geológicas desarrolladas por erosión glacial, son usualmente alongados, profundos y de paredes abruptas (a menudo, más profundos que la plataforma continental adyacente); típicamente poseen uno o más umbrales (morrenas) que definen las cuencas profundas del fiordo y usualmente hay un río descargando en la cabeza de éste (Freeland et al. 1980, Farmer \& Freeland 1983, Dyer 1997).

La circulación de los fiordos está determinada por una serie de procesos, dentro de los cuales destaca la mezcla diapicna, flujos a través de estrechos, salto hidráulico y forzamientos baroclinos y barotrópicos. En particular, los principales procesos que determinan el intercambio horizontal corresponden a la circulación intermedia y circulación estuarina (Aure \& Stigebrandt 1990, Stigebrandt 1990, 2001, Aure et al. 1997). La circulación intermedia está asociada al gradiente de densidad horizontal existente entre el fiordo y la zona costera adyacente. La circulación estuarina, por otra parte, está asociada a la relación entre la descarga de agua dulce, el 'entrainment' y la influencia del viento sobre el proceso de mezcla. Esta circulación ha sido típicamente descrita como una estructura de 2 capas, una capa superficial con flujo de salida hacia al mar, y una capa inferior con un flujo neto de ingreso hacia el fiordo. Sin embargo, estudios recientes indican que esta estructura es más compleja, con 
una circulación en 3 capas (Cáceres et al. 2010, Castillo et al. 2012, 2016; Valle-Levinson et al. 2014), lo cual ha sido también demostrado a través de modelos analíticos (Winant 2008).

La zona de fiordos del sur de Chile ha sido sistemáticamente estudiada durante los últimos 20 años, lo que ha permitido describir la distribución horizontal y vertical de las principales variables oceanográficas físicas y químicas (Sievers \& Prado 1994, Silva et al. 1995, 1997, 1998; Nimpscht 2000, Dávila et al. 2002, Guzmán 2004, Calvete 2006, Silva \& Guzmán 2006, Calvete \& Sobarzo 2011). Recientemente, los estudios se han enfocado en describir los procesos dinámicos de intercambio en fiordos y canales del sur de Chile, concentrándose principalmente en los flujos residuales (ValleLevinson et al. 2001, Cáceres et al. 2002a, b; Valle-Levinson et al. 2002a, Cáceres 2004, Cáceres \& Valle-Levinson 2004, Salinas \& Hormazábal 2004, Salcedo-Castro 2006).

Uno de los sistemas más estudiados, en la extensa zona de fiordos y canales del sur de Chile, es el fiordo Aysén. En este fiordo, Cáceres et al. (2002a, 2002b) y Valle-Levinson et al. (2002b) proponen un esquema de circulación de 3 capas, con un tercer flujo neto de salida en la capa de fondo. Esta dinámica ha sido corroborada por estudios recientes en los fiordos Aysén y Reloncaví (Cáceres et al. 2010, Castillo et al. 2012, 2016; Valle-Levinson et al. 2014), a través de mediciones con ADCP remolcado. Sin embargo, la circulación residual en el fiordo Aysén, así como esta estructura de 3 capas, no han sido estudiadas mediante series de tiempo. Por lo tanto, el objetivo del presente estudio fue describir la circulación residual en el fiordo Aysén, mediante el análisis de una serie de tiempo de 3 correntómetros instalados en su boca, considerando el efecto del viento, gasto del río Aysén y salinidad. La hipótesis de trabajo fue que la estructura de 3 capas propuesta para este sistema es un rasgo permanente y que el viento es un forzante relevante en su dinámica.

\section{Materiales Y MÉTODOS}

El fiordo Aysén ( $\left.45^{\circ} 20^{\prime} \mathrm{S} ; 7^{\circ} 05^{\prime} \mathrm{W}\right)$, tiene una orientación general E-W, desde la cabeza a la boca, orientando su eje longitudinal en sentido SE-NW en los primeros dos tercios del fiordo, y reorientándose en sentido NE-SW más hacia la boca, donde se localiza la isla Elena, la cual separa la boca del fiordo del canal Pilcomayo. Este canal presenta una zona muy somera $(<50 \mathrm{~m})$ en la entrada que comunica con el fiordo (Fig. 1).

Se realizaron mediciones de corrientes en la boca del fiordo Aysén, en un anclaje de correntómetros ubicados a 6, 50 y $110 \mathrm{~m}$ de profundidad, desde el 5 de octubre al 8 de noviembre de 1996 (Fig. 1). En el nivel de $6 \mathrm{~m}$ se instaló un correntómetro Aanderaa RCM-7, con sensores de velocidad (magnitud y dirección), temperatura y salinidad. En los niveles de 50 y $110 \mathrm{~m}$ se instalaron correntómetros Sensordata SD6000. Los instrumentos fueron configurados con un intervalo de registro cada $10 \mathrm{~min}$.

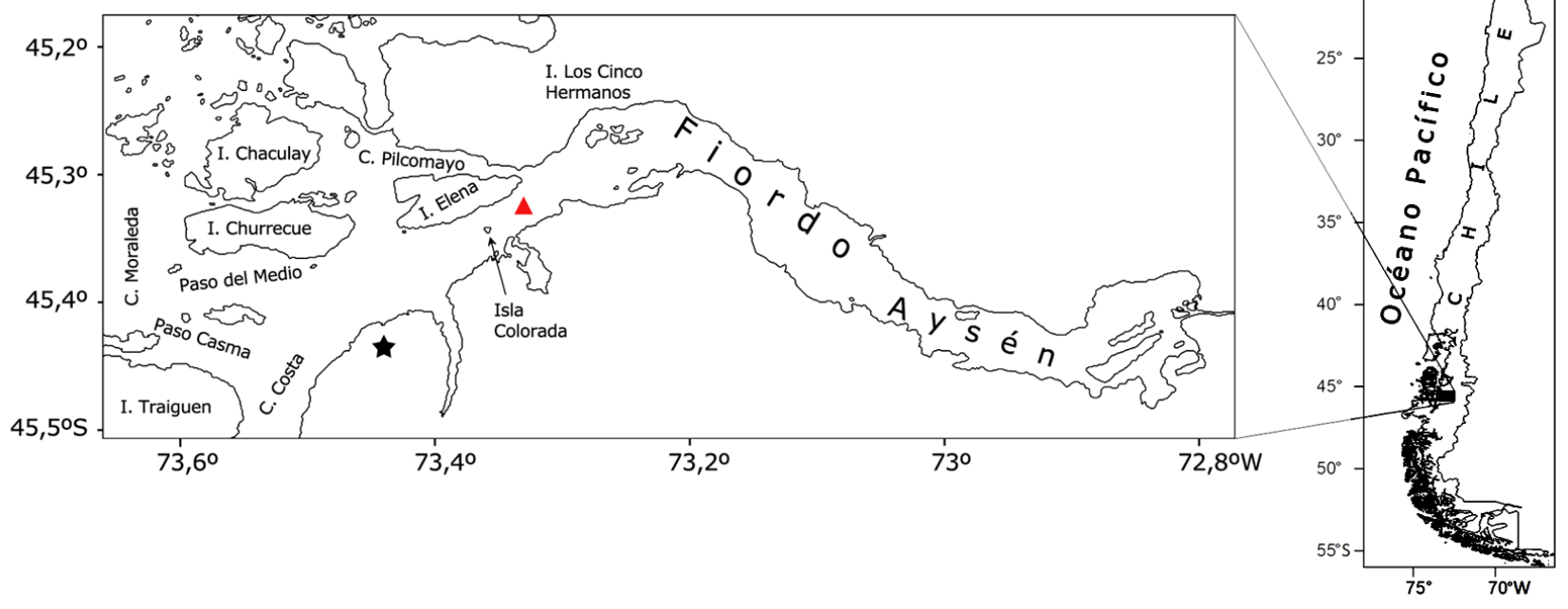

Figura 1. Localización del anclaje en la boca del fiordo Aysén (triángulo rojo), donde se instalaron correntómetros a 6,50 y $110 \mathrm{~m}$ de profundidad. La estrella representa el punto correspondiente a la serie de viento obtenida del reanálisis CFSR / Location of mooring at the mouth of Aysén fjord (red triangle), where current meters were installed at 6, 50 y $110 \mathrm{~m}$ depth. The star represents the point corresponding to the wind series obtained from CFSR reanalysis 
En cada una de las series de tiempo, los componentes ortogonales del vector de velocidad, $u(\mathrm{~N}-\mathrm{S})$ y $v(\mathrm{O}-\mathrm{E})$, fueron rotados de manera que la componente $u$ coincidiera con el eje del fiordo e incluyera así la máxima variabilidad del flujo (Preisendorfer \& Mobley 1988, Emery \& Thomson 1998). La componente $u$ representó así el flujo longitudinal del fiordo y la componente $v$, el flujo transversal.

A las series de corrientes se les extrajo la componente de marea, usando el paquete UTide para MATLAB (Codiga 2011). Posteriormente, a la serie residual se le aplicó un filtro de paso bajo Butterworth de $12 \mathrm{~h}$. El mismo filtro, fue aplicado para las series de corrientes, temperatura y salinidad.

Debido a la inexistencia de registros meteorológicos en la zona, se obtuvo una serie de tiempo del viento desde National Centers for Environmental Prediction (NCEP) Climate Forecast System Reanalysis (CFSR) (Saha et al. 2010), con la finalidad de evaluar el forzamiento del viento sobre la circulación del fiordo. Las coordenadas de este punto se indican en la Fig. 1. Por otra parte, para realizar una comparación de los diferentes forzantes del sistema, se obtuvieron los registros de caudales medios diarios del río Aysén, proporcionados por la Dirección General de Aguas (DGA).

A la serie de viento obtenida de CFSR (originalmente, generada con un intervalo cada $6 \mathrm{~h}$ ) se le aplicó un proceso de interpolación para generar una nueva base de datos con un intervalo cada $10 \mathrm{~min}$. De esta manera se pudo realizar un análisis de correlación cruzada con las respectivas series de velocidad de la corriente. Igual metodología fue utilizada para la serie de tiempo del caudal del río Aysén. Por otra parte, se realizó un análisis integrado de la variación temporal del flujo de la corriente en los 3 niveles de medición, relacionándolos con la variación del caudal del río Aysén y el forzante viento, durante el periodo de las mediciones.

\section{Resultados}

En la Figura 2 se indican las series temporales de los vectores de viento (Fig. 2a), la salinidad a $6 \mathrm{~m}$ de profundidad junto con el caudal del río Aysén (Fig. 2b) y la componente residual $u$ rotada en la dirección del eje del fiordo en las 3 profundidades (Figs. 2c, d y e).

La variación de las corrientes observadas en el anclaje de correntómetros en la boca del fiordo Aysén mostró amplias diferencias entre las 3 profundidades, como se observa en las series respectivas (Fig. 2). El correntómetro situado a $6 \mathrm{~m}$ exhibió mayores magnitudes de velocidad, presentando eventos de fuerte flujo de salida (valores negativos) que se prolongaron por 2 a 3 días y que coincidieron con descenso en la salinidad superficial (Fig. 2b y c). Durante estos eventos de intenso flujo superficial de salida, el correntómetro de 110 m mostró un similar al observado en la serie de $6 \mathrm{~m}$, aunque en dirección al interior del fiordo (valores positivos) y con un rango menor de magnitudes de velocidad (Fig. 2e). En contraste a lo observado en los niveles de $6 \mathrm{~m}$ y $110 \mathrm{~m}$, a 50 $\mathrm{m}$ se observó una tendencia poco definida que muestra procesos de transición entre flujos de entrada y salida.

El análisis de la matriz de coeficientes de correlación para las series de corrientes, salinidad superficial, viento y caudal del río Aysén se muestra en la Tabla 1. Solo se consideró la componente N-S del viento (componente $v$ ), ya que arrojó coeficientes de correlación más significativos. Consistente con los resultados en la Fig. 2, los coeficientes muestran eventos desfasados de reflujo en superficie, que corresponden a flujo en la capa profunda y viceversa; la correlación entre la componente $u$ a $6 \mathrm{~m}$ y $110 \mathrm{~m}$ es de $-0,169(P<0,05)$. Resultados dinámicamente importantes los representa la correlación entre la componente del $u$ a $6 \mathrm{~m}$ y la salinidad. La influencia del viento en la salinidad superficial y la componente $u$ a $6 \mathrm{~m}$ se refleja en la correlación negativa de $-0,606$ y $0,629(P<0,05)$, respectivamente.

Tabla 1. Coeficientes de correlación cruzada entre los componentes longitudinales de velocidad a 6,50 y $110 \mathrm{~m}$, respecto al caudal del río, viento (componente N-S) y los valores de salinidad superficial / Cross-correlation coefficients among the longitudinal velocity components at 6,50 and $110 \mathrm{~m}$, in relation to river discharge, wind (N-S component) and surface salinity

\begin{tabular}{crrrrrr}
\hline & CompU6m & CompU50m & CompU120m & Sal6m & $\begin{array}{r}\text { Viento } \\
\text { CompV }\end{array}$ & Caudal \\
\hline CompU6m & 1,000 & & & & & \\
CompU55m & 0,002 & 1,000 & & & & \\
CompU120m & $-0,169$ & $-0,103$ & 1,000 & & & \\
Sal6m & 0,984 & 0,018 & $-0,197$ & 1,000 & & \\
Viento CompV & $-0,605$ & 0,042 & 0,204 & $-0,629$ & 1,000 & \\
Caudal & $-0,119$ & $-0,346$ & $-0,167$ & $-0,128$ & $-0,135$ & 1,000 \\
\hline
\end{tabular}



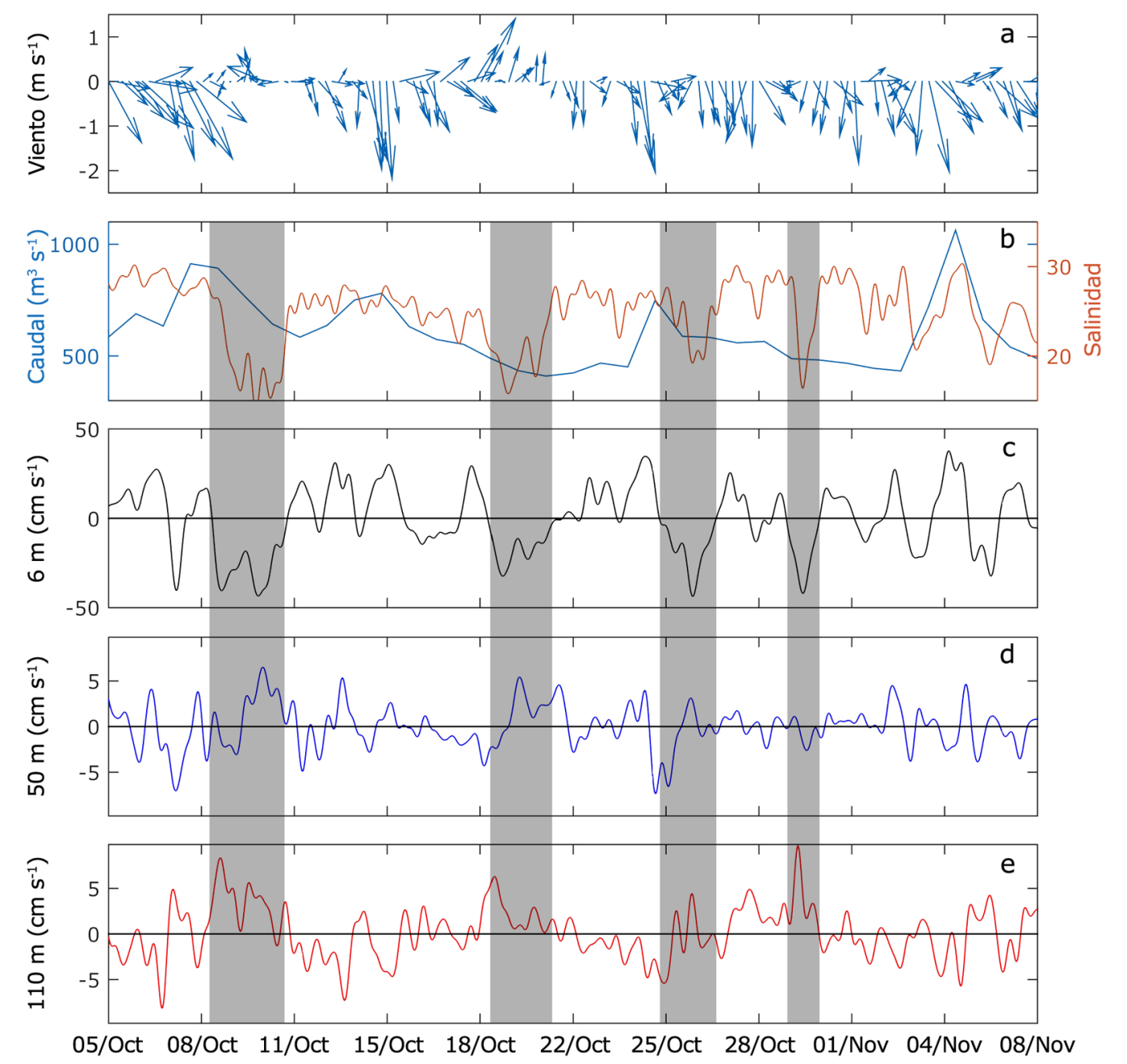

Figura 2. Variación temporal de (a) vectores de viento en el sector del fiordo Aysén (ver Fig. 1), (b) descarga del río Aysén y
valores superficiales de salinidad y (c-d-e) magnitud de la componente rotada de corrientes a 6, 50 y 110 m de profundidad en
la boca del fiordo Aysén. Los valores positivos indican corriente hacia la cabeza del fiordo. Se muestran en sombreado los
episodios de mayor flujo y menor salinidad superficial (ver texto). / Temporal variation of (a) wind vector in the area of Aysén
fjord (See Fig. 1), (b) Aysén river discharge and surface salinity, and (c-d-e) magnitude of the rotated current component at 6,50
and $110 \mathrm{~m}$ depth at the mouth of Aysén fjord. Positive values indicate up-fjord current. Episodes of strong surface flux and
lower salinity are shown in grey (see text)

En la serie correspondiente a $6 \mathrm{~m}$, la magnitud de la corriente varió en un rango de 0 a $64 \mathrm{~cm} \mathrm{~s}^{-1}$, mostrando eventos de amplias y rápidas variaciones del flujo, principalmente de salida. La dirección del flujo estuvo predominantemente dirigida hacia afuera del fiordo, lo cual se tradujo en un flujo promedio de $-9,64 \mathrm{~cm} \mathrm{~s}^{-1}$ (Tabla 2). El registro de corrientes obtenido a $50 \mathrm{~m}$ de profundidad presentó corrientes que no superaron $\operatorname{los} 10 \mathrm{~cm} \mathrm{~s}^{-1}$. Sin embargo, la corriente no presentó una tendencia definida de ingreso o salida del fiordo, arrojando sólo una corriente promedio de -
$0,07 \mathrm{~cm} \mathrm{~s}^{-1}$ (Tabla 2). A $110 \mathrm{~m}$ de profundidad, la velocidad de las corrientes fue mayor que aquella observada a $50 \mathrm{~m}$, con magnitudes de hasta $24,78 \mathrm{~cm} \mathrm{~s}^{-1}$. Esta serie mostró una asociación más notoria con el registro de $6 \mathrm{~m}$ que la observada en la serie de $50 \mathrm{~m}$ (Tabla 1). El flujo promedio obtenido a esta profundidad fue de $-1,04 \mathrm{~cm} \mathrm{~s}^{-1}$ (Tabla 2), indicando un flujo neto de salida desde el fiordo. Un perfil estimado a partir de las velocidades medias de las series de corrientes registradas a 6,50 y $110 \mathrm{~m}$ es presentado en la Figura 3. 
Tabla 2. Corriente promedio y valores máximo y mínimo de corrientes de entrada y salida al fiordo Aysén / Average velocity and maximal and minimal outflow/inflow current in Aysén fjord

\begin{tabular}{cccc}
\hline $\begin{array}{c}\text { Profundidad } \\
(\mathrm{m})\end{array}$ & $\begin{array}{c}\text { Velocidad media } \\
\left(\mathrm{cm} \mathrm{s}^{-1}\right)\end{array}$ & $\begin{array}{c}\text { Máxima velocidad } \\
\text { de entrada } \\
\left(\mathrm{cm} \mathrm{s}^{-1}\right)\end{array}$ & $\begin{array}{c}\text { Máxima velocidad } \\
\text { de salida } \\
\left(\mathrm{cm} \mathrm{s}^{-1}\right)\end{array}$ \\
\hline 6 & $-9,64$ & 56,22 & $-64,68$ \\
50 & $-0,07$ & 19,90 & $-17,90$ \\
110 & $-1,04$ & 24,78 & $-20,48$ \\
\hline
\end{tabular}

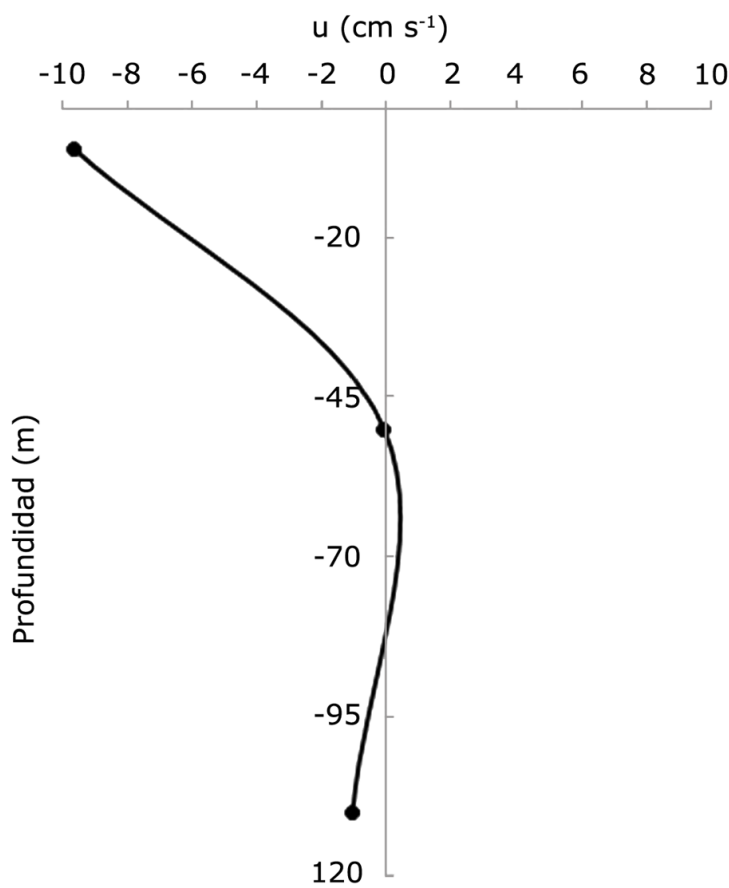

Figura 3. Perfil de las velocidades promedio (u) a 6, 50 y $110 \mathrm{~m}$ en la boca del fiordo Aysén / Profile of average velocities (u) at 6, 50 and $110 \mathrm{~m}$ depth at the mouth of Aysén fjord

Se observa una débil influencia del caudal del río Aysén sobre la variabilidad de la corriente y salinidad superficial (Fig. $2 \mathrm{~b}$ y c). Sin embargo, el aumento del caudal del río puede haber sido un factor importante en la intensificación del flujo superficial de salida (valores negativos) observado en la boca del fiordo, con posterioridad a los días 8 y 25 de octubre y, en menor medida, el 5 de noviembre (Fig. 2 b y c). La magnitud y dirección de la corriente superficial presentó una correlación significativa $(P<0,05)$ con la salinidad de este estrato (Tabla 1), lo que se observó claramente al comparar las fluctuaciones de ambas variables (Fig. 2b y c). Esto indica la predominancia de un proceso advectivo por sobre un proceso de mezcla.

\section{Discusión}

El análisis de la correntometría en la boca del fiordo Aysén mostró un flujo superficial promedio de salida, mientras que a $50 \mathrm{~m}$ de profundidad, las magnitudes, aunque bajas, presentaron la misma dirección en el flujo medio. Por otra parte, a $110 \mathrm{~m}$ se observó un flujo medio de mayor magnitud que a $55 \mathrm{~m}$, también en dirección hacia afuera del fiordo (Tabla 2, Fig. 3). Al respecto, Cáceres et al. (2002b), en un experimento realizado al sureste de isla Elena, sobre un bajo situado al noreste de isla Colorada, observaron una estructura de 3 capas, con una capa superficial $(<8 \mathrm{~m})$ de salida $(3 \mathrm{~cm}$ $\mathrm{s}^{-1}$ ) (extrapolado, no resuelto por el instrumento), una región intermedia (30 a $40 \mathrm{~m}$ de grosor) de ingreso $\left(10 \mathrm{~cm} \mathrm{~s}^{-1}\right.$ ), asociada al viento $\mathrm{SW}$ predominante durante el experimento, y una región profunda (60-110 m de grosor) con un flujo compensatorio de salida $\left(5 \mathrm{~cm} \mathrm{~s}^{-1}\right)$. Los flujos observados a $6 \mathrm{~m}$ y $110 \mathrm{~m}$ podrían corresponder a la capa superficial y profunda, respectivamente, descrita por estos autores, mientras que las observaciones obtenidas a $50 \mathrm{~m}$, dados los bajos valores registrados, parecen corresponder a una zona de transición entre las capas intermedia y profunda. Así, la capa intermedia del flujo residual no habría sido capturada en su parte central sino en el límite superior durante este estudio, como indica el esquema de la Fig. 3. La presencia de estas 3 capas también fue observada por Cáceres (2004) en la boca del fiordo, describiendo un flujo superficial de salida (primeros $15 \mathrm{~m}$ ), un segundo flujo de ingreso que se extendió entre $15 \mathrm{y}$ $70 \mathrm{~m}$, atribuido al forzamiento de la marea, y una tercera capa profunda, bajo los $70 \mathrm{~m}$, probablemente producida por un gradiente de presión longitudinal generado por el viento soplando hacia la cabeza del fiordo. En este caso, la zona de transición entre la capa intermedia y la profunda también se ubicaría alrededor de los 60-70 m.

Notablemente, la serie de velocidades de corrientes a 6 $\mathrm{m}$, muestra un importante efecto del viento, reflejado en el coeficiente de correlación negativa. Durante el periodo de estudio, el viento predominante se dirigió hacia el SE, imponiendo una mayor resistencia al flujo superficial. Este efecto opuesto queda en evidencia cuando ocurre un relajamiento del viento, al aumentar el flujo superficial, junto con una disminución de la salinidad, lo que indica un flujo de la capa superficial que se ve favorecido, mientras el ingreso en la capa de fondo se acentúa (Fig. 2). Este rol del viento en la circulación de los fiordos del sur de Chile ha sido destacado previamente. Por ejemplo, Valle-Levinson et al. (2002b) describieron la existencia de un flujo hacia el interior del fiordo cerca de la superficie (bajo los $8 \mathrm{~m}$ de profundidad) y un flujo hacia el canal Costa en una capa inferior, señalando que la 
capa superficial estuvo impulsada por los vientos predominantes (WSW) durante el periodo de muestreo. La capa intermedia, con un grosor de 60-100 m, probablemente habría sido forzada por un gradiente barotrópico de presión, asociado al desnivel del mar producido por el viento. Trabajando sobre un sistema cercano, en la constricción de Meninea, Valle-Levinson et al. (2002a) propusieron una explicación similar, asociada al efecto del viento. Estos investigadores señalan que el intercambio fue más intenso durante periodos con vientos débiles. Hubo eventos asociados a viento norte intenso $\left(>10 \mathrm{~cm} \mathrm{~s}^{-1}\right)$, en los cuales se revirtieron los flujos o se debilitaron, y se profundizó la interface entre ambos flujos. El viento norte tendió a oponerse al flujo de salida inducido por densidad y originó una pendiente negativa del nivel del mar (decreciendo hacia el norte). Este gradiente de presión barotrópico produciría un flujo (de salida) cercano al fondo que también se opone al flujo de entrada inducido por densidad. Por lo tanto, el viento norte intenso debilitaría ambos flujos y podría llegar a crear una estructura de flujo de tres capas: una superficial de ingreso, intermedia de salida y una tercera de ingreso. Al debilitarse el viento, la capa superficial intensificaría su flujo de salida hacia el norte, mientras la capa de fondo intensificaría su flujo hacia el sur. En esta misma área, Salinas \& Hormazábal (2004) instalaron un anclaje y registraron velocidades promedio de $15,9 \mathrm{~cm} \mathrm{~s}^{-}$ ${ }^{1}$ hacia el norte $(25 \mathrm{~m})$ y $2,0 \mathrm{~cm} \mathrm{~s}^{-1}$ hacia el sur $(40 \mathrm{~m})$, comparables a los obtenidos en este estudio. Además, estos autores establecen que las corrientes están dominadas por la componente semidiurna $\mathrm{M}_{2}$, observando una alternancia de flujos de características barotrópicas (marea) y baroclinas (estuarino).

El perfil obtenido a partir de las velocidades medias apoya la idea la existencia de una estructura de 3 capas propuesta por Cáceres et al. (2010) y Valle-Levinson et al. (2014) y que es similar a la descrita por Castillo et al. $(2012,2016)$ en el fiordo Reloncaví. En particular, el correntómetro ubicado a $55 \mathrm{~m}$ presenta un muy débil flujo residual que indicaría la zona de transición entre las capas media y profunda que describen estos autores.

Los resultados del flujo residual observado en los datos de correntometría confirman la presencia de una tercera capa con un flujo de salida cercana al fondo. Esta confirmación, con una serie de datos más larga que la presentada por Cáceres et al. (2002a, b) y Cáceres (2004), pone en evidencia este rasgo poco estudiado en los sistemas de fiordos y destaca el fuerte rol del viento como modulador de la circulación en fiordos del sur de Chile, incluso, por sobre la influencia de la magnitud de las descargas de agua dulce en estos sistemas.

\section{Agradecimientos}

Los autores agradecen las sugerencias y comentarios de los revisores anónimos, los cuales ayudaron a mejorar y enriquecer este trabajo. El segundo autor agradece al Dr. Guillermo Gutiérrez de Velazco Sanromán por su ayuda con el procesamiento de los datos.

\section{LITERATURA CITADA}

Aure J \& A Stigebrandt. 1990. Quantitative estimates of the eutrophication effects of fish farming on fjords. Aquaculture 90: 135-156.

Aure J, J Molvaer \& A Stigebrandt. 1997. Observations of inshore water exchange forced by a fluctuating offshore density field. Marine Pollution Bulletin 33: 112-119.

Cáceres M. 2004. Surface features of the circulation in fjords of southern Chile observed in ERS and LANDSAT images. Gayana 68(2) Suppl. 1: 71-76.

Cáceres M \& A Valle-Levinson. 2004. Transverse variability of flow on both sides of a sill/contraction combination in a fjord-like inlet of southern Chile. Estuarine Coastal and Shelf Science 60: 325-338.

Cáceres M, A Valle-Levinson, J Fierro, C Valenzuela \& M Castillo. 2002a. Variabilidad transversal del flujo y de la densidad en la boca del Seno Aysén. En: Libro de Resúmenes, Taller Sobre los Resultados del Crucero CIMAR 7 Fiordos, pp. 45-47. CONA,Valparaíso.

Cáceres M, A Valle-Levinson, H Sepúlveda \& $\mathrm{K}$ Holderied. 2002b. Transverse variability of flow and density in a Chilean fjord. Continental Shelf Research 22: 1683-1698.

Cáceres M, A Valle-Levinson, J Fierro, C Valenzuela \& M Castillo. 2010. Variabilidad transversal del flujo y de la densidad en la boca del Fiordo Aysén. Ciencia y Tecnología del Mar 33(1): 5-15.

Calvete C. 2006. Caracterización de zonas ROFI y frentes asociados en los fiordos del sur de Chile ubicados entre la boca del Guafo y laguna San Rafael. Tesis para Magíster en Ciencias, Mención en Oceanografía, Universidad de Concepción, Concepción, 80 pp.

Calvete C \& M Sobarzo. 2011. Quantification of the surface brackish water layer and frontal zones in southern Chilean fjords between Boca del Guafo (43 $30^{\circ}$ 'S) and Estero Elefantes (46³0’S). Continental Shelf Research 31: 162171.

Castillo M, O Pizarro, U Cifuentes, N Ramírez \& L Djurfeldt. 2012. Subtidal dynamics in a deep fjord of southern Chile. Continental Shelf Research 49: 73-89.

Castillo M, U Cifuentes, O Pizarro, L Djurfeldt \& M Cáceres. 2016. Seasonal hydrography and surface outflow in a fjord with a deep sill: The Reloncaví fjord, Chile. Ocean Science 12: 533-544. 
Codiga DL. 2011. Unified tidal analysis and prediction using the UTide Matlab Functions. In: Technical Report 2011-01, Graduate School of Oceanography, University of Rhode Island, Narragansett, 59 pp.

Dávila P, D Figueroa \& E Müller. 2002. Freshwater input into the coastal ocean and its relation with the salinity distribution off austral Chile $\left(35-55^{\circ} \mathrm{S}\right)$. Continental Shelf Research 22: 521-534.

Dyer K. 1997. Estuaries a physical introduction, 195 pp. John Wiley \& Sons, Chichester.

Emery WJ \& RE Thomson. 1998. Data analysis methods in physical oceanography, 634 pp. Pergamon Press, Oxford.

Farmer D \& H Freeland. 1983. The physical oceanography of Fjords. Progress in Oceanography 12(2): 147-220.

Freeland H, D Farmer \& C Levings. 1980. Fjord Oceanography. NATO Conference Series. Series IV. Marine Sciences (NATO) 4: 1-715. Plenum Press, New York.

Guzmán D. 2004. Caracterización hidrográfica, oceanográfica y balance de nitrógeno y fósforo del fiordo Aysén. Tesis para Magíster en Oceanografía, Pontificia Universidad Católica de Valparaíso, Valparaíso, 74 pp.

Preisendorfer RW \& CD Mobley. 1988. Principal component analysis in meteorology and oceanography. Developments in Atmospheric Science 17: 1-425 Elsevier Science, New York.

Saha S, S Moorthi, H-L Pan, X Wu, JJ Wang, S Nadiga, $P$ Tripp, R Kistler, J Woollen, D Behringer, H Liu, D Stokes, R Grumbine, G Gayno, JJ Wang, Y-T Hou, HY Chuang, H-M Juang, J Sela, M Iredell, R Treadon, D Kleist, P Van Delst, D Keyser, J Derber, M Ek, J Meng, H Wei, R Yang, S Lord, H Van Den Dool, A Kumar, W Wang, C Long, M Chelliah, Y Xue, B Huang, J-K Schemm, W Ebisuzaki, R Lin, P Xie, M Chen, S Zhou, W Higgins, C-Z Zou, Q Liu, Y Chen, Y Han, L Cucurull, RW Reynolds, G Rutledge \& M Goldberg. 2010. The NCEP climate forecast system reanalysis. Bulletin of the American Meteorological Society 91(8): 1015-1057.

Salcedo-Castro J. 2006. Oceanografía del fiordo Aysén y flujos residuales en su boca. Tesis de Magister en Oceanografía, Universidad de Valparaíso, Valparaíso 74 pp.

Salinas S \& S Hormazábal. 2004. Capacidad de transporte de la constricción de Meninea para un flujo de dos capas y el efecto de la corriente de marea. Ciencia y Tecnología del Mar 27(1): 5- 15.

Sievers H \& R Prado. 1994. Contraste de las características oceanográficas del seno Aysén, Chile, entre invierno y verano (Lat. 45²0’S). Revista de Biología Marina 29(2): 167-209.
Silva N \& D Guzmán. 2006. Condiciones oceanográficas físicas y químicas, entre boca del Guafo y fiordo Aysén (Crucero Cimar 7 Fiordos). Ciencia y Tecnología del Mar 29(1): 25 44.

Silva N, H Sievers \& R Prado. 1995. Características oceanográficas y una proposición de circulación, para algunos canales australes de Chile entre $41^{\circ} 20^{\prime} \mathrm{S}$ y $46^{\circ} 40^{\prime} \mathrm{S}$. Revista de Biología Marina 30(2): 207-254.

Silva N, C Calvete \& H Sievers. 1997. Características oceanográficas físicas y químicas de canales australes chilenos entre Puerto Montt y Laguna San Rafael (Crucero CIMAR-Fiordo 1). Ciencia y Tecnología del Mar 20: 23 106

Silva N, C Calvete \& H Sievers. 1998. Características Masas de agua y circulación general para algunos canales australes canales entre Puerto Montt y Laguna San Rafael (Crucero CIMAR-Fiordo 1). Ciencia y Tecnología del Mar 21: 17-48.

Stigebrandt A. 1990. On the response of the horizontal mean vertical density distribution in a fjord to low-frequency density fluctuations in the coastal water. Tellus 42A: 605-614.

Stigebrandt A. 2001. FJORDENV-a water quality model for fjordsand other inshore waters, 41 pp. Earth Sciences Centre, Göteborg University, Göteborg.

Valle-Levinson A, F Jara, C Molinet \& D Soto. 2001. Observations of intratidal variability of flows over a sill/ contraction combination in a Chilean fjord. Journal of Geophysical Research 106(C4): 7051-7064.

Valle-Levinson A, J Blanco \& J Fierro. 2002a. Observations of wind effects on exchange flows in a channel constriction of the Chilean Inland Sea. In: The 2nd Meeting on the Physical Oceanography of Sea Straits, Villefranche, 15th-19th April 2002, pp. 223-227.

Valle-Levinson A, M Cáceres, H Sepúlveda \& K Holderied. 2002b. Patrones de flujo en los canales asociados a la boca del seno Aysén. Ciencia y Tecnología del Mar 25(2): 5-16.

Valle-Levinson A, M Cáceres \& O Pizarro. 2014. Variations of tidally driven three-layer residual circulation in fjords. Ocean Dynamics. 64: 459-469.

Winant CD. 2008. Three-dimensional residual tidal circulation in an elongated, rotating basin. Journal of Physical Oceanography 38 : 1278-1295. 